International Journal of Engineering \& Technology, $7(1.2)(2018) 181-185$
International Journal of Engineering \& Technology
WPC
Website: www.sciencepubco.com/index.php/IJET
Research paper

\title{
A novel implementation of backpressure algorithm in wireless ad hoc network
}

\author{
Ugendhar Addagatla $^{1 *}$, V. Janaki ${ }^{2}$ \\ ${ }^{1}$ Department of C.S.E, Guru Nanak Institutions Technical Campus, Ranga Reddy, Telangana-501506 \\ ${ }^{2}$ Department of C.S.E, Vaagdevi Engineering College, Warangal, Telangana-506005 \\ *Corresponding author E-mail: ugendhar2009@gmail.com
}

\begin{abstract}
In the wireless networks, the routing technique is the one of the highest concern and it is the important procedure in the ad hoc networks. To aid this effort, we proposed an new valuation of backpressure appliances for wireless networks. By this proposed system, we will address numerous preparation and routing difficulties and also recover the throughput and delay that are essentially produced by the packets at the node transmission. The Backpressure routing is a dense and enlarged throughput for wireless networks, but endures improved delays. In routing, the backpressure algorithm is known to afford throughput optimality with active traffic. The significant supposition in the backpressure algorithm is that all nodes are kind and detect the algorithm rules leading the information conversation and principal optimization necessities. In the proposed system, we validate that how the node is steady at the backpressure algorithm routing and also by together easing the virtual trust line and the real package queue. The backpressure algorithm not only achieves flexibility, but also stands the throughput performance under safety attacks. This scheme is mostly enhances the node performance at the time of announcement and also it recovers the node security at the time of numerous threats in the wireless requests.
\end{abstract}

Keywords: Backpressure Algorithm; Throughput Optimality; Dynamic Traffic; Node Transmission.

\section{Introduction}

In this algorithm needs remained reflected as an effective line length-based arrangement and routing example since its presence. It has concerned a lot of consideration due to its notable advantages, e.g., throughput optimality (i.e. It can stabilize a network when the entrance rates lie within the capacity area of the network), achievable adaptive resource allocation, support to stateless and agile load-aware routing and scheduling, and easiness. Newly, a lot of work has been approved out, and much development needs remained complete to recover the performance of back-pressure preparation in dissimilar system surroundings [1]. Though, the poor interruption performance of back-pressure algorithm is one of the key difficulties delaying its wide arrangement in repetition. To ease the understanding, in here, we concisely announce how back-pressure preparation works as follows. Backpressure algorithm needs each network node to keep a queue for individually flow traversing it (namely, per-flow queue). At each time slot, it works to stimulate a set of non-interference relations in the network (i.e., it chooses a set of non-interference links for communicating packets), which mains to the extreme sum of link weights multiplying their equivalent link rates, to transmit packets [2]. The link weight connected with a link is defined as the main flow weight on the link, where the movement weight on a link is the queue accumulation difference for the movement between the two endpoints of the link.

However, such a scheduling strategy can frequently cause huge latency, which is often accredited to the subsequent three details [3]. First, back-pressure-based scheduling typically suffers from slow startup phenomenon. That is, when a movement starts, numerous packets of it have to be backlogged on the method to ter- minus to form unchanging queue backlog-based incline, which reductions towards the destination, for supporting smooth packet delivery to the destination. Such slow startup reasons huge primary end-to end (E2E) packet delay [4].

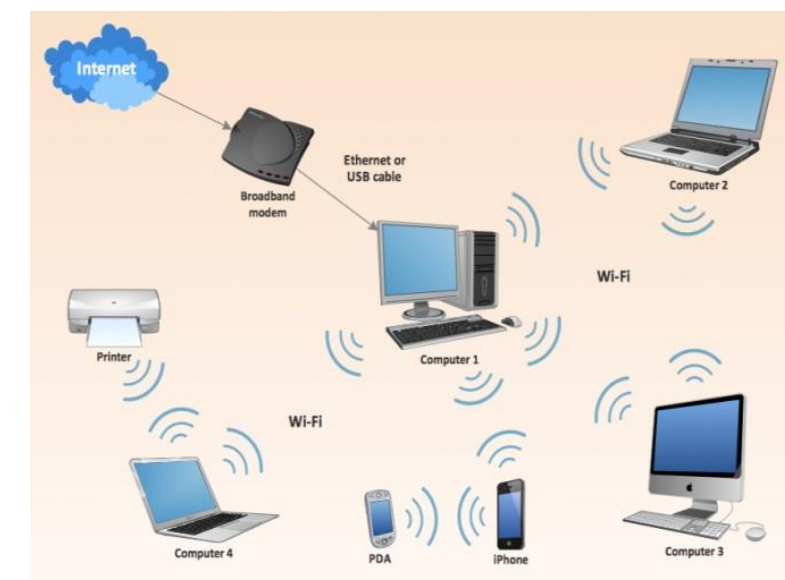

Fig. 1: Ad Hoc Wireless Network.

Another, variation of the queue backlog-based gradient in the setting of back-pressure scheduling frequently reasons data packets to take needlessly extended or even looped pathways, which largely raises the packet interruption. Third, the so-called lastpacket problematic can also reason huge potential in networks with short-lived or low-rate movements due to the absence of dependable back-pressure near the destination [5].

To discourse the exceeding problems, in this paper, we initiative a back-pressure routing and preparation algorithm for wireless sen- 
sor networks. VBR is planned to pre-establish a simulated queue based gradient for nodes in a WSN. In VBR, this algorithm-based preparation is achieved conferring to the queue distance which is the amount of actual queue length and virtual queue length at separately node. The incline related with a node is a purpose of traffic entrance rate, network link rate, and its distance to sink, in instruction to be adaptive to dissimilar network and application surroundings while attaining high packet delivery performance [6], [7] Verify that VBR preserves back-pressure's throughput optimality. Simulation consequence displays that VBR can get important presentation development in terms of packet distribution relation, average E2E delay, and regular per-node line length as linked with existing work [8].

The presentation of backpressure disparages in conditions of low, and reasonable in the network, i.e., this algorithm improves the system using all conceivable paths all over the network. The opposing consequence of this algorithm is to increase interruption and also to growth the energy consumption of the nodes. This is because of End to end delay and energy ingesting is dependable [9]. The minimization of the normal time that the packets stay in the system proposes a reduction in the normal number of hops that the packs travel until they inspiration their endpoint, which in turn proposes a reduction in the whole energy consumption.

\section{Back pressure routing}

Backpressure routing mentions to an algorithm for enthusiastically routing traffic ended a multi-hop network by using congestion ascents. It typically mentions to a data network, but can apply to other kinds of networks as well. Under we attention on the data network submission, where numerous data streams reach to a network and important be dispersed to appropriate terminuses. The backpressure algorithm purposes in positioned time, and each slot it follows to route data in instructions that exploit the differential backlog among neighboring nodes [10], [11].

This is parallel to how water would movement finished a network of pipes via pressure gradients. Though, the backpressure algorithm can be functional to multi-commodity networks and to networks where transmission rates can be designated from dissimilar (possibly time varying) choices. Attractive features of the backpressure algorithm are:

i). It mains to extreme network throughput

ii). It is provably vigorous to time-varying network situations.

iii). It can be executed without significant traffic entrance charges or channel state probabilities.

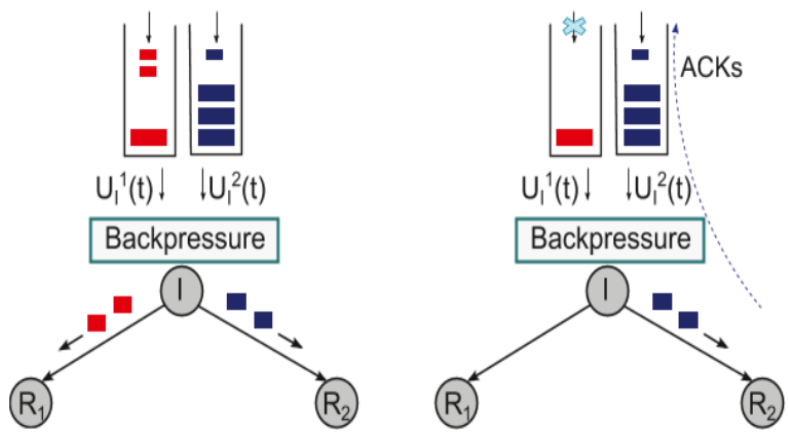

Fig. 2: An Example of Backpressure Routing.

a) Backpressure with random arrivals

b) Back pressure with TCP arrivals

\section{Proposed system}

\subsection{Backpressure algorithm}

The backpressure algorithm is the perfect response that requires essential organization. In reality, an integrated controller determination gathers information from all nodes then sort the preparation choice. There likewise occurs low-complexity, transmitter answers with presentation close to the greatest solution $[13,14]$. The algorithm continues a queue for each endpoint at each node. Since the amount of destinations can be as large as the amount of nodes, this per-destination queuing condition can be rather huge for practical execution in a network. At each link, the algorithm allocates a weight to each conceivable destination which is called backpressure. Define the backpressure at link (nj) for destination $\mathrm{d}$ at slot t to be

$W^{d}{ }_{n j}[t]=Q_{n d}[t]-Q_{j d}[t]$,

Where $\mathrm{Q}_{\mathrm{nd}}[\mathrm{t}]$ signifies the amount of packages at node $\mathrm{n}$ intended for node $\mathrm{d}$ at the establishment of time slot $\mathrm{t}$. Under this notation, $\mathrm{Q}_{\mathrm{nn}}[\mathrm{t}]=0, \forall \mathrm{t}$. Allocate a weight wnj to each link (nj),

Where $w_{n j}$ is defined to be the maximum back-pressure over all possible destinations, i.e.,

$W_{n j}[t]=\max _{d} W^{d}{ }_{n j}[t]$.

Let $\mathrm{d} * \mathrm{nj}$ be the destination which has the maximum weight on link (nj),

$\mathrm{d}^{*}{ }_{\mathrm{nj}}[\mathrm{t}]=\arg \max _{\mathrm{d}}\left\{\mathrm{w}^{\mathrm{d}} \mathrm{nj}[\mathrm{t}]\right\}$.

If there are ties in the weights, they can be broken arbitrarily. Packets belonging to destination $\mathrm{d} * \mathrm{nj}[\mathrm{t}]$ are scheduled for transmission over the activated link (nj).

\subsection{Threats in backpressure algorithm}

In common, the performance of an insider attacker can be categorized to one or both of the following two groups.

- Information-falsification attack: this occurs through the information dispute phase at the initial of individually time slot, where the attacker decisively directs false information to others to unenviable affect this routing.

- As the algorithm is receptive to node queue bottlenecks and station national information, its routing outcomes can be suggestively precious by information-falsification attacks.

- Protocol-violation attack: this happens in the prescribed transmission stage, where the aggressor prepares not submit this routing decisions.

\subsection{Rate control and utility maximization}

Further newly, usefulness expansion has been addressed in a backpressure outline to address rate control problems. We use the preparation in to designate the idea here. Each flow $f[s, d]$ creating from $\mathrm{s}$ and intended for $\mathrm{d}$ has a efficacy function Uf $[\mathrm{s}, \mathrm{d}](\mathrm{xf}[\mathrm{s}, \mathrm{d}])$ which is a purpose of the rate $\mathrm{xf}[\mathrm{s}, \mathrm{d}]$ it is attended at. Let sf and $\mathrm{df}$ represent the source and the endpoint of the flow f, correspondingly. We accept that all usefulness purposes are severely concave, with unceasing derivatives. The usefulness expansion problem is the following:

$\max \Sigma_{\mathrm{f} \in \mathrm{F}} \mathrm{u}_{\mathrm{xf}}(\mathrm{x}$ f $)$

Let $\mathrm{xf}[\mathrm{t}]$ signify the rate at which the flow $\mathrm{f}$ is served in time slot t. The rate control algorithm that exploits is the following. In respectively time slot $t$, the source sf injects $\kappa>0$ packets into the queue $\mathrm{q} \mathrm{df}$ sf if and only if

$U^{\prime} f\left(x_{f}[t]\right)-\beta q_{s}{ }_{f}^{d} f[t]>0$,

Where $\beta>0$ is a device parameter and $\mathrm{U}^{\prime} \mathrm{f}$ is the primary derived of flow f's usefulness purpose. The parameter $\beta$ controls how close to the best rate distribution the scheme achieves, but this comes at the price of longer queues [15]. 


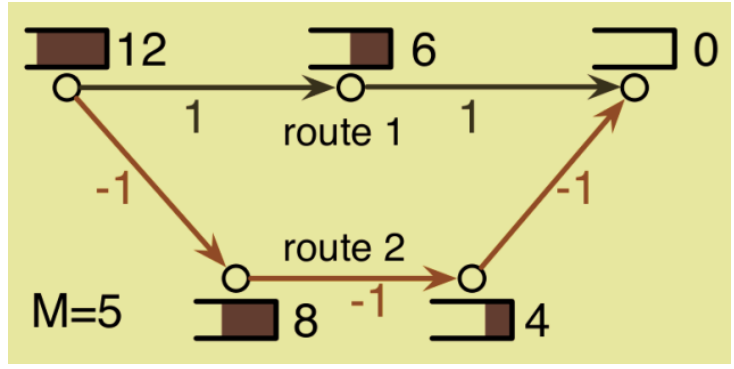

Fig. 3: Back Pressure Algorithm.

\section{Performance evaluations}

The performance assessment, an extensive simulation study is to estimate the performance of the proposed secure backpressure algorithm in a node. The arrangement of the wireless network contains 50 nodes with distribution range $80 \mathrm{~m}$ reliably spread over quantified area. The protocol interfering model is accepted. Furthermore, if a node is getting from a neighbor at a time slot, none of its other neighbors will be scheduled to communicate. We deliberate a whole set of attack circumstances in the models:

1) Black hole attacks is the attacks in which it continuously broadcast zero queue backlogs and high frequency rates to fascinate packets to be directed to them, then drop all received packets.
2) On-off attacks in which perform as black holes or sincere nodes during on and off periods.

3) Selfish nodes always challenge to empty its queues by propagating high queue backlogs to detention the transmission opportunity.

4) Heterogeneous attacks include all above attackers at different nodes in the same network.

In the performance assessment, we designate the metric of throughput as the normal quantity of distributed data per time slot regularized by the connection rate. The back-pressure algorithm, though being throughput best, is not useful in practice for adaptive routing since the interruption performance can be actually bad. In this paper, we have obtainable an algorithm that routes packets on through hops when possible, and decouples routing and preparation using a probabilistic excruciating algorithm built on the concept of shadow queues. By preserving a probabilistic routing table that variations gradually over time, real packets do not must to discover long paths to recover throughput, this functionality is achieved by the shadow "packets." Our algorithm also allows extra link initiation to decrease delays. The algorithm has also remained shown to decrease the queuing complexity at separately node and can be protracted to optimally tradeoff among routing and network coding [16]

0.8

0.6

$\begin{array}{ll}\text { 蒿 } & 0.4 \\ \text { b0 } & \\ 0 & \\ 0 & \\ 0 & \end{array}$

0.2

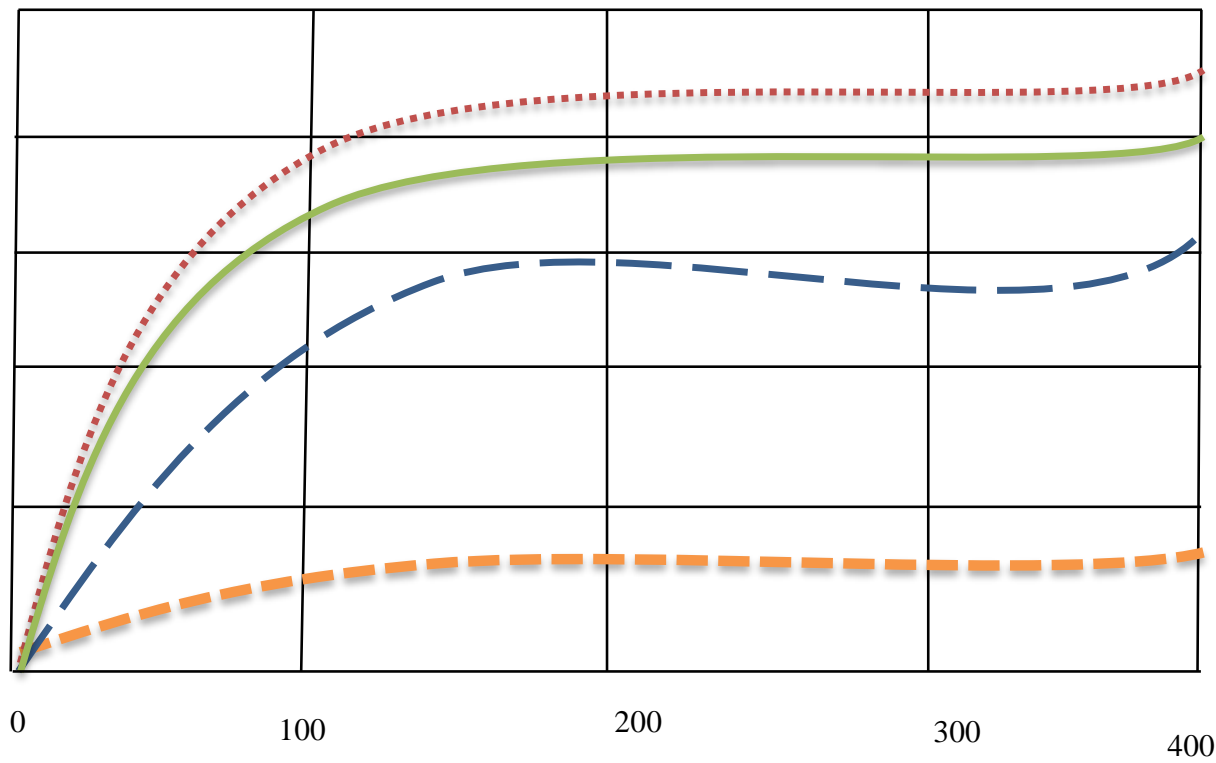

Runtime

Fig. 4: Throughput over Run Time for Different Scenarios.

No attack

Low tolerance trust
High tolerance trust

No defense under attack 


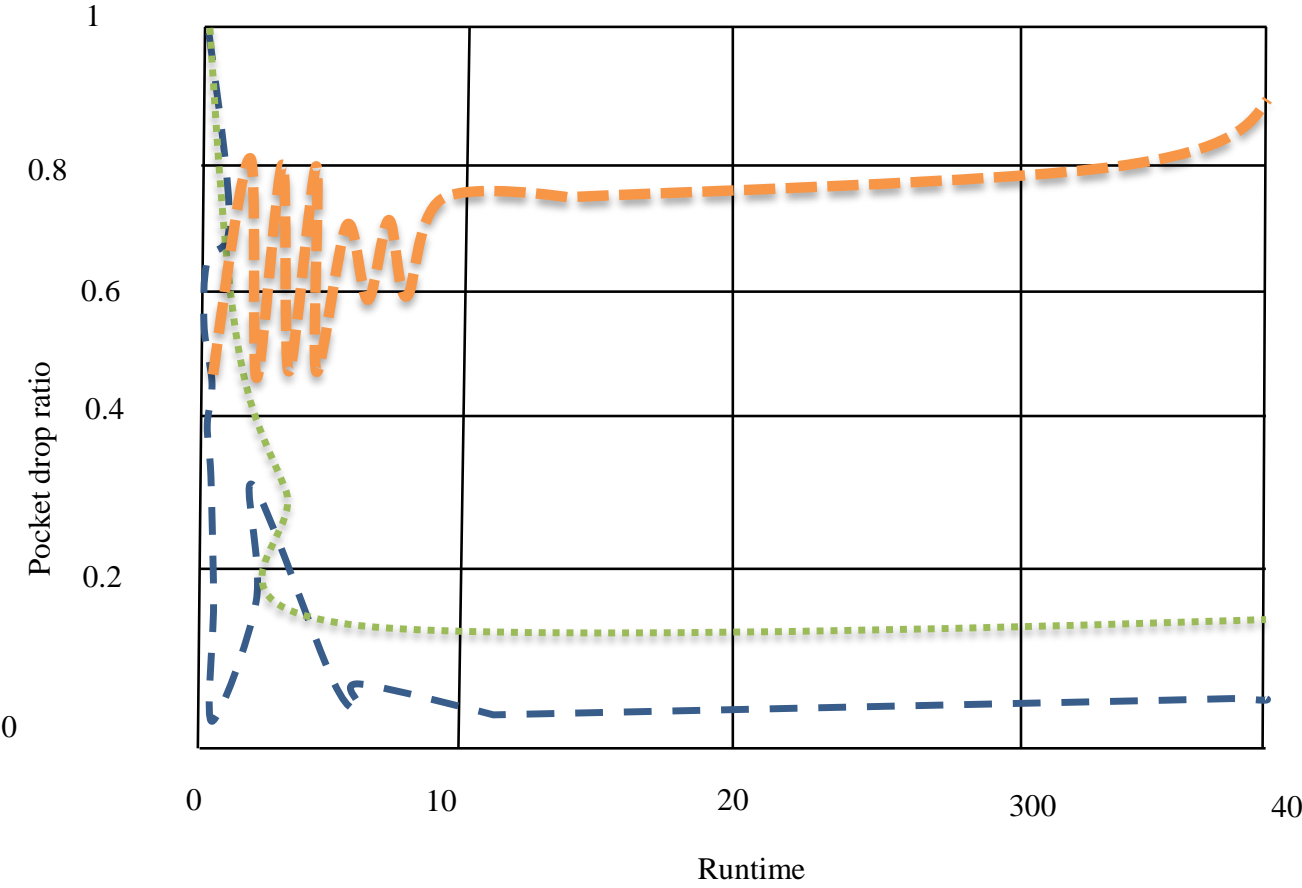

Fig. 5: Packet Drop Ratio over Run Time for Different Scenarios.

In simulation procedure, randomly choice one node in the network performance as a black hole. Fig.4establishes the throughput in the network below the black hole attack. Firstly, the network throughput increases as run time permits. This is because the network is informally loaded in the early state. As additional packets spread at each node, the network throughput growths increasingly and becomes constant. However, when there is an attacker, we can see over $85 \%$ deficiency of the throughput in the network. The output of the scheme is essentially essences on the refining competence. If an attacker stimulates outside the given acceptance level, which results in an unbalanced queue, the attacker will be excluded from the routing decision. Fig. 5illustrates the packet drop ratio in network under the same attacks. We detect from the figure, the packet drop ratio is zero lacking the occurrence. Hence, in the proposed system, throughput is increased and the packet drop ratio is decreased [17], [18].

\section{Conclusion}

In this proposed system, that providing an capable work on the algorithm at the node level and likewise recover the security of the system. Finally, we presented complete simulations to validate the competence of the recommended mechanism. The consequences showed that the simulated trust line mechanism obtains the algorithm in inconsistency of a wide range of attacks. Accordingly, the solution from this proposed system disperses a main barrier for applied preparation of backpressure algorithm for endangered wireless applications. Hence, the algorithm not only attains flexibility, but also suffers the throughput presentation under security attacks. This arrangement is usually recovers the node performance at the time of communication and also it developments the node safety at the time of numerous threats in the wireless applications

\section{References}

[1] Maglaras, L.A. and Katsaros, D. (2011) Layered backpressure scheduling for delay reduction in ad hoc networks. In World of Wireless, Mobile and Multimedia Networks (WoWMoM), 2011 IEEE International Symposium on a (IEEE): 1-9. https://doi.org/10.1109/WoWMoM.2011.5986388.
[2] Gupta, G. and Shroff, N. (2009) Delay analysis for multi-hop wireless networks. In INFOCOM 2009, IEEE: 2356-2364 https://doi.org/10.1109/INFCOM.2009.5062162.

[3] A. Warrier, S. Janakiraman, S. Ha, and I. Rhee, "DiffQ: Practical differential backlog congestion control for wireless networks," inProc. of IEEE INFOCOM, 2009.

[4] F. Bao, I.-R. Chen, M. Chang, and J.-H Cho, "Hierarchical trust management for wireless sensor networks and its applications to trust-based routing and intrusion detection," vIEEE Trans. Network and Service Management, vol. 9, pp. 169-183, Mar. 2012. https://doi.org/10.1109/TCOMM.2012.031912.110179.

[5] L. Georgiadis, M. J. Neely, and L. Tassiulas, "Resource allocation and cross-layer control in wireless networks," Foundations and Trends in Networking, vol. 1, pp. 1-144, 2006. https://doi.org/10.1561/1300000001.

[6] H. Seferoglu and E. Modiano, "Diff-Max: Separation of routingand scheduling in backpressure-based wireless networks," in Proc.of IEEE INFOCOM, 2013.

[7] S. Moeller, A. Sridharan, B.Krishnamachari, and O. Gnawali Routing without routes: The backpressure collection protocolProc. $9^{\text {th }}$ ACM/IEEE Intl. Conf. on Information Processing in Sensor Networks (IPSN), April 2010.

[8] L. Huang, S. Moeller, M. J. Neely, and B. Krishnamachari. LIFObackpressure achieves near optimal utility-delay tradeoff. Proc. WiOpt, May 2011.

[9] L. Huang, S. Moeller, M. J. Neely, and B. Krishnamachari,"LIFObackpressure achieves near optimal utility-delay tradeoff,"ACM/IEEE Trans. Networking, pp. 831-844, June 2013.

[10] L. Bui, R. Srikant, and A. L. Stolyar, "A novel architecture for delay reduction in the back-pressure scheduling algorithm," IEEE/ACM Trans. Netw., vol. 19, no. 6, pp. 1597-1609, Dec. 2011. https://doi.org/10.1109/TNET.2011.2126593.

[11] M. Alresaini, M. Sathiamoorthy, B. Krishnamachari, and M. J. Neely,"Backpressure with adaptive redundancy (BWAR)," in Proc. of IEEE INFOCOM, 2012 https://doi.org/10.1109/INFCOM.2012.6195617.

[12] J. Nunez-Martinez, J. Mangues-Bafalluy, and M. Portoles-Comeras, "Studying practical any-to-any backpressure routing inWi-Fi mesh networks from a Lyapunovoptimization perspective,"in Proc. of IEEE MASS, 2011

[13] Bui, L., Srikant, R. and Stolyar, A. (2009) Novel architectures and algorithms for delay reduction in back- pressure scheduling and routing. In INFOCOM 2009, IEEE (IEEE): 2936-2940. https://doi.org/10.1109/INFCOM.2009.5062262.

[14] B. Radunovic, C. Gkantsidis, D. Gunawardena, and P. Key. Horizon: Balancing TCP over multiple paths in wireless mesh networkACMMobicom, 2008.

[15] Ying, L., Srikant, R., Towsley, D. and Liu, S.(2011) Cluster-based back-pressure routing algorithm. Networking, IEEE/ACM Transac- 
$\begin{array}{lll}\text { tions } & \text { on } & 19(6) \text { : } \\ \text { https://doi.org/10.1109/TNET.2011.2141682. }\end{array}$

1773-1786.

[16] J.-Y. Yoo, C. Sengul, R. Merz, and J. Kim, "Experimental analysisof backpressure scheduling in IEEE 802.11 wireless meshnetworks," in Proc. of IEEE ICC, 2011.

[17] Li, R., Eryilmaz, A. and Li, B. (2013) Throughput optimal wireless scheduling with regulated inter-servicetimes. In INFOCOM, 2013 Proceedings IEEE: https://doi.org/10.1109/INFCOM.2013.6567069.

[18] L. Ying, S. Shakkottai, A. Reddy, and S. Liu,"On combiningshortest-path and back-pressure routing over multihop wirelessnetworks," IEEE/ACM Trans. Networking, vol. 19, Jun 2011. https://doi.org/10.1109/TNET.2010.2094204.

[19] S. Liu, L. Ying, and R. Srikant,"Throughput-optimal opportunisticscheduling in the presence of flow-level dynamics,' IEEE/ACMTrans. Networking, vol. 19, Aug 2011. https://doi.org/10.1109/TNET.2010.2100826.

[20] L. Bui, R. Srikant, and A. L. Stolyar, "Optimal resource allocation for multicast flows in multihop wireless networks," Phil. Trans. Roy. Soc., Ser.A, vol. 366, pp. 2059-2074, 2008. 